\title{
POTENTIAL IMPROVEMENT OF AGRICULTURAL OUTPUT FOR MAJOR PRODUCERS BASED ON DEA EFFICIENCY MEASUREMENTS
}

\author{
Geraldo da Silva e Souza ${ }^{1}$, Tito Belchior Silva Moreira ${ }^{2}$ \\ and Eliane Gonçalves Gomes ${ }^{3 *}$
}

Received November 2009 / Accepted August 2010

\begin{abstract}
In this article we perform production efficiency analysis for the 40 countries with largest value added by agricultural sector in 2005 . Under the assumption of a nonparametric frontier and production observations satisfying a statistical model including both random and inefficiency errors, we estimate an agricultural production function using DEA measures of efficiency with output orientation and variable returns to scale. We found evidence that the set of countries investigated could increase their total value added by agricultural sector for at least $53.9 \%$ without increasing input usage with the prevailing technology. This result has a direct impact on issues related to the recent food crisis.
\end{abstract}

Keywords: food crisis, efficiency, DEA, agriculture.

\section{INTRODUCTION}

The world has been affected lately (2006 to 2008) by dramatic rises in food prices, generating a global crisis and causing political and economical instability and social unrest in both poor and developed nations.

Systemic causes for the worldwide increases in food prices continue to be the subject of debate. Initial causes of the late 2006 price spikes includes unseasonable droughts in grain producing nations and rising oil prices. Oil prices further heightened the costs of fertilizers, food transport, and industrial agriculture. Other causes may be the increasing use of biofuels in developed countries and an increasing demand for a more varied diet (especially meat) across the expanding middle-class populations of Asia. These factors, coupled with falling world food stockpiles, have all contributed to the dramatic worldwide rise in food prices. However, to explain the recent crisis, it is not possible to elect a specific guilty.

\footnotetext{
*Corresponding author

${ }^{1}$ Brazilian Agricultural Research Corporation (Embrapa), Parque Estação Biológica, Av. W3 Norte final, $70770-901$ Brasília, DF, Brazil. E-mail: geraldo.souza@embrapa.br

${ }^{2}$ Catholic University of Brasilia, SGAN 916, Módulo B, 70790-160 Brasília, DF, Brazil. E-mail: tito@pos.ucb.br

3 Brazilian Agricultural Research Corporation (Embrapa), Parque Estação Biológica, Av. W3 Norte final, $70770-901$ Brasília, DF, Brazil. E-mail: eliane.gomes@embrapa.br
} 
Long-term causes remain a topic of debate. These may include structural changes in trade and agricultural production, agricultural price supports and subsidies in developed nations, diversions of food commodities to high input foods and fuel, commodity market speculation, and climate change. In this context it is worth mentioning Nicholson \& Esseks (1978), Dyson (1994), Food and Agriculture Organization of the United Nations (2008a), Organization for Economic Co-operation and Development (2008), OXFAM International (2008), Rosegrant (2008), World Bank (2008a, b), World Economic Forum (2008), International Food Policy Research Institute (2008), Abbott et al. (2008), Asian Development Bank (2008), Dawe (2008), Ivanic \& Martin (2008), Valdés \& Foster (2008), and Von Braun et al. (2008). Other lines of research use total factor productivity indexes to investigate the effects of contextual variables. Examples are Fulginiti \& Perrin (1997), Nin et al. (2003) and Thirtle et al. (2003).

Our main interest is not to investigate the causes of the food crisis, but the assessment of the actual world potential to increase the supply of agricultural goods. In this context we use a new Data Envelopment Analysis - DEA approach based on the work of Banker \& Natarajan (2004, 2008) in the presence of contextual variables. Using projections onto the frontier, with possible corrections for random effects, we show that the food crisis can be minored substantially if the economies become more efficient relative to the technology available. Hence, this article has two main contributions: a new approach for the assessment of contextual variables using two stage DEA models incorporating two error components, and a suggestion of a security food policy via reduction of production inefficiencies.

This paper does not intend to propose solutions for the food crisis. Instead it provides a diagnostic that may be useful for policymakers to propose measures contributing to agricultural development via incentive policies to reduce the inefficiency of production of agricultural goods. To resolve the food crisis we understand that it is necessary a coordination of global policies to reduce the scarcity of food. In this context firstly it is necessary to define a rough diagnosis of the potential capacity of world producers.

Based on methods of efficiency analysis it is possible to rank countries via efficiency scores. The score of each country is the ratio of the frontier output to actual output. The frontier output is not necessarily achievable, since there are difficulties to compare countries with different technologies, land qualities, labor, capital etc. However, projections on the frontier are useful to pinpoint countries where, in a first approximation, it would be possible to increase production without demanding additional inputs. For example, we will see in this paper that many large producers of agricultural goods are classified as very inefficient, i.e., with a score of efficiency lower than the median. This result suggests that the agricultural production of these countries can be substantially improved, reducing substantially the world agricultural output gap.

The potential capacity is the output projection onto the frontier, and the output gap is the difference between the frontier output and current agricultural production. The efficiency frontier is a proxy for the world agricultural potential capacity, despite of all restrictions on estimation due to aggregation across countries. 
Once potential inefficiencies have been identified, policy makers may provide a closer look on inefficient producers with the intent to create mechanism designs envisaging those producers to become more efficient.

In the literature there are several studies using efficiency analysis and output frontier across countries with the intent to provide support for prescriptions of public policies.

Arcellus \& Arocena (2005) carry out a computational analysis of the tradeoffs between a good output and the $\mathrm{CO}_{2}$ emissions or bad output of the production process across OCDE countries via a DEA formulation. The authors compute potential frontier $\mathrm{CO}_{2}$ reduction and compare their results with targets defined by the Kyoto protocol emission limitations. Based on their findings they provide possible negotiation strategies for the various countries in their effort to reach a pollution control agreement.

Tyagi et al. (2009) evaluate the performance of 19 academic departments of different areas, in India. They use DEA and suggest the improvement of performance via projections on the input and output oriented frontiers.

Sharma \& Thomas (2008) examine the relative efficiency of the R\&D process across a group of 22 developed and developing countries using DEA. The R\&D technical efficiency is examined using a model with patents granted to residents as an output and gross domestic expenditures on R\&D and the number of researchers as inputs. The emergence of some of the developing nations on the efficiency frontier indicates that these nations can also serve as benchmarks for their efficient use of R\&D resources. The inefficiency in the R\&D resources usage highlighted by this study indicates the underlying potential that can be tapped for the development and growth nations.

We follow here a similar line of investigation. We define the problem, use a method of efficiency analysis, compute the efficient frontier, point out the inefficient DMUs, and provide helpful information to policymakers to identify which countries can improve their agricultural production performance via inefficiencies reduction.

The Brazilian literature on the subject of measuring agricultural efficiency at world level is scarce, as described in Gomes (2008). On this theme, we can cite the papers of Headey et al. (2010), Reimer \& Kang (2010), Rezitis (2010), Coelli \& Rao (2005), Gorton \& Davidova (2004), Thiam et al. (2001), for example. There are some important studies related to agricultural productivity at region, state and county levels in Brazil. In this context it is worth to mention the works of Souza et al. (2010), Nogueira (2005), Baptista et al. (2004), Vicente (2004), Otsuki et al. (2002), Pereira et al. (2002), among others.

The article proceeds as follows. Section 2 is on methodological aspects, where we specify the statistical model and the selection of participating countries. Section 3 analyzes efficiency and statistical results and proposes a world policy increase in agricultural supply. In Section 4 we present final comments and summarize the main findings of the article. 


\section{METHODOLOGICAL ASPECTS}

\subsection{Output, Inputs and Contextual Variables}

The countries considered in this article are listed in Table 1 . They comprise a universe of the 40 countries with the largest value added by agricultural sector. Together they were responsible, in 2005 , for roughly $80 \%$ of the world agricultural sector.

The production system in our analysis involves one output and four inputs. As a proxy for the agricultural output we use value added by the agricultural sector, in 2005 , in $10^{9}$ dollars at constant prices. Value added is the net output of a sector, after adding up all outputs and subtracting intermediate inputs. This information is available in World Bank (2008c).

Inputs are land, labor, fertilizers and capital. The source for the input data is Food and Agriculture Organization of the United Nations (2008b).

For land we use agricultural area, which refers to: (a) arable land (land under temporary crops, temporary meadows for mowing or pasture, land under market and kitchen gardens and land temporarily fallow, (b) permanent crops (land cultivated with crops that occupy the land for long periods and need not be replanted after each harvest, and (c) permanent pastures (land used permanently for herbaceous forage crops, either cultivated or growing wild (wild prairie or grazing land). Data are expressed in 1,000 hectares. We follow Coelli \& Rao (2005).

The economic active population in agriculture defines labor. This variable is defined as the agricultural labor force, i.e., that part of the economically active population engaged in or seeking work in agriculture, hunting, fishing or forestry. Data are expressed in 1,000 people, in 2005. We follow Fulginiti \& Perrin (1997) and Coelli \& Rao (2005).

For fertilizer we used the total fertilizer consumption, in 2005. It's the quantity of fertilizer consumed in agriculture expressed in tonnes of plant nutrients. It's represented by the sum of the consumptions of nitrogen ( $\mathrm{N}$ total nutrients), phosphate $\left(\mathrm{P}_{2} \mathrm{O}_{5}\right.$ total nutrients) and potash $\left(\mathrm{K}_{2} \mathrm{O}\right.$ total nutrients). This procedure is in accordance with Food and Agriculture Organization's statistics regarding fertilizers consumption. Data are expressed in tonnes of nutrients. We follow the studies of Hayami \& Ruttan (1970), Fulginiti \& Perrin (1997) and Coelli \& Rao (2005).

As a proxy for capital we use the capital stock in agriculture that refers to a value that is attached to the total physical capital capacity available for repeated use in the production of other goods, in existence at specific point in time in the economy of agriculture sector. As stated in Food and Agricultural Organization of the United Nations (2008b), the estimates of investment in agriculture have indirectly been derived by the FAO Statistics Division using physical data on livestock, tractors, irrigated land and land under permanent crops etc., and the average prices for the year 1995. These data enabled the derivation of the capital stock in agriculture which is the gross; the annual change in the latter is taken to reflect investment in agriculture. Capital expenses are not available for 2005. The most recent estimate for capital available in our sources 
is for 2003. As a proxy for capital in 2005 we take the ratio capital/output observed in 2003 and multiply it by the output level in 2005. Data are expressed in US\$ at constant 1995 price.

Production data is shown in Table 1, where HDI and R1, R2, R3, R4, R5, R6, R7 appear as contextual variables. HDI is the Human Development Index (United Nations Development Programme, 2006), a proxy for income and development. The HDI is taken for the year 2004 to avoid contemporaneous correlation with 2005 residuals. R1-R7 are dummies representing countries' geographical regions, as in World Bank (2008c).

Some interesting approaches integrating HDI and DEA may be seen in Mahlberg \& Obersteiner (2001) and Despotis (2005a, b). Specifically using HDI as a contextual variable we cite the work of Antunes et al. (2006).

The approach employed here uses a production function with output and input aggregations by countries to perform production efficiency analysis. This approach is commonly used in the literature as can be seen in Ray \& Desli (1997), Maudos et al. (1999), Kumar \& Russell (2002), and Arcelus \& Arocena (2005).

The raw data was screened for the presence of outliers using regression methods as follows. Let $w=\left(1, y, x_{1}, x_{2}, x_{3}, x_{4}, R_{1}, \ldots, R_{6}\right)$ be the matrix formed with observations on output $y$ and inputs $x_{i}$ plus a column of ones and regional dummies. It is a standard procedure in regression analysis (Kutner et al., 2004) to consider values greater than two times the average of the diagonal elements of the matrix $w\left(w^{\prime} w\right)^{-1} w^{\prime}$ as outlying observations. Such observations were identified by a dummy contextual variable 'outlier'. These were Australia, China, Japan and United States. This variable did not show statistical significance in the second stage.

\subsection{Statistical Production Model}

The production analysis is carried out considering a nonparametric model. We assume that observations on production follow the statistical model (1),

$$
y_{j}=g\left(x_{j}\right)+v_{j}-u_{j} \quad j=1 \ldots n
$$

where $g(\cdot)$ is a continuous production function defined on the compact convex set $K$ in the nonnegative orthant of $R^{4}$, with nonempty interior, satisfying:

1. $x, w \in K, \quad \forall t \in[0,1], \quad \operatorname{tg}(x)+(1-t) g(w) \leq g(t x+(1-t) w)$.

2. $x, w \in K, \quad x \geq w, \quad g(x) \geq g(w)$.

3. $g(\cdot)$ shows variable returns to scale.

The random variables $v_{j}$ and $u_{j}$ represent random and inefficiency errors respectively. Following Banker \& Natarajan $(2004,2008)$ we assume that the random errors have a two sided 


\begin{tabular}{|c|c|c|c|c|c|c|c|c|c|c|c|c|c|c|c|c|c|c|c|}
\hline$\widehat{\approx}$ & 0 & 0 & 0 & 0 & 0 & 0 & 0 & 0 & 0 & 0 & 0 & 0 & 0 & 0 & 0 & 0 & 0 & 0 & 0 \\
\hline$\approx$ & 0 & 0 & 0 & - & 0 & 0 & 0 & 0 & 0 & 0 & 0 & 0 & 0 & - & 0 & 0 & 0 & 0 & 0 \\
\hline$\approx$ & 0 & 0 & 0 & 0 & 0 & - & 0 & 0 & 0 & 0 & 0 & 0 & 0 & 0 & 0 & 0 & 0 & 0 & 0 \\
\hline$\stackrel{ \pm}{\simeq}$ & -1 & 0 & 0 & 0 & 0 & 0 & 0 & 0 & 0 & - & 0 & 0 & 0 & 0 & 0 & - & 0 & 0 & 0 \\
\hline$\tilde{\simeq}$ & 0 & - & 0 & 0 & - & 0 & - & 0 & - & 0 & 0 & 0 & 0 & 0 & 0 & 0 & 0 & 0 & 0 \\
\hline 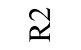 & 0 & 0 & 0 & 0 & 0 & 0 & 0 & 0 & 0 & 0 & - & - & - & 0 & 0 & 0 & - & 0 & 0 \\
\hline $\bar{\simeq}$ & 0 & 0 & - & 0 & 0 & 0 & 0 & - & 0 & 0 & 0 & 0 & 0 & 0 & -1 & 0 & 0 & - & - \\
\hline 宔 & $\left|\begin{array}{c}\infty \\
\stackrel{N}{0} \\
0^{\prime}\end{array}\right|$ & $\begin{array}{l}m \\
\infty \\
0 \\
0\end{array}$ & $\begin{array}{l}\hat{n} \\
\hat{\sigma} \\
0\end{array}$ & $\begin{array}{l}0 \\
\tilde{n} \\
n \\
0\end{array}$ & $\mid \begin{array}{l}2 \\
\hat{\Omega} \\
0\end{array}$ & $\begin{array}{l}\text { ڤn } \\
\hat{\sigma}\end{array}$ & $\begin{array}{l}2 \\
\hat{\delta} \\
\infty \\
0\end{array}$ & $\left.\mid \begin{array}{l}\infty \\
0 \\
0 \\
0\end{array}\right]$ & $\frac{2}{2}$ & 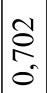 & $\begin{array}{c}\mathcal{y} \\
\mathscr{L} \\
0\end{array}$ & $\begin{array}{l}\tilde{\sigma} \\
\hat{\sigma}\end{array}$ & $\begin{array}{l}\overrightarrow{\widehat{\sigma}} \\
\sigma_{0}\end{array}$ & $\begin{array}{l}\bar{\sigma} \\
\overline{0} \\
0\end{array}$ & $\vec{\nabla}$ & $\begin{array}{l}\frac{1}{n} \\
0 \\
0\end{array}$ & $\mid \begin{array}{l}\mathcal{1} \\
\dot{a} \\
0\end{array}$ & $\begin{array}{l}\hat{\sigma} \\
\text { o }\end{array}$ & $\frac{\Im}{\sigma}$ \\
\hline 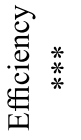 & \begin{tabular}{|l}
8 \\
8 \\
8 \\
- \\
-1
\end{tabular} & 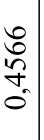 & $\begin{array}{c}\hat{2} \\
\hat{2} \\
\hat{0}\end{array}$ & $\begin{array}{c}0 \\
\tilde{\infty} \\
\infty \\
\tilde{m} \\
0\end{array}$ & 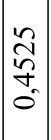 & \& & $\mid \begin{array}{l}\infty \\
2 \\
\infty \\
\tilde{0} \\
0\end{array}$ & $\begin{array}{l}8 \\
0 \\
8 \\
0 \\
-1\end{array}$ & $\begin{array}{l}\tilde{0} \\
\tilde{\sigma} \\
0 \\
0\end{array}$ & $\begin{array}{l}2 \\
\hat{5} \\
\vdots \\
0 \\
0 \\
0\end{array}$ & $\begin{array}{l}8 \\
8 \\
8 \\
-1 \\
-1\end{array}$ & $\mid \begin{array}{l}n \\
\infty \\
0 \\
0 \\
0 \\
0\end{array}$ & $\begin{array}{l}8 \\
8 \\
8 \\
-\end{array}$ & $\mid \begin{array}{c}\hat{\partial} \\
\tilde{m} \\
\hat{0} \\
0\end{array}$ & $\begin{array}{c}\infty \\
m \\
\\
\vdots \\
0\end{array}$ & 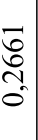 & $\begin{array}{l}\infty \\
\tilde{N} \\
\hat{\sigma} \\
0 \\
0\end{array}$ & $\begin{array}{l}8 \\
8 \\
0\end{array}$ & 8 \\
\hline 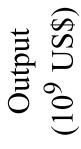 & 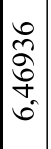 & $\begin{array}{l}8 \\
8 \\
n \\
n \\
n \\
n\end{array}$ & $\begin{array}{l}\hat{\Xi} \\
\overline{0} \\
\dot{\Xi}\end{array}$ & $\begin{array}{l}\Xi \\
\Xi \\
\Xi \\
\ddot{\Xi}\end{array}$ & 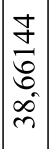 & 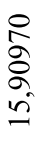 & $\begin{array}{l}\frac{N}{J} \\
\frac{\pi}{N} \\
i n\end{array}$ & $\mid \begin{array}{c}8 \\
\infty \\
\infty \\
n \\
n \\
\\
\end{array}$ & $\begin{array}{l}n \\
\infty \\
\infty \\
\tilde{b} \\
0 \\
0\end{array}$ & $\left|\begin{array}{l}\infty \\
2 \\
8 \\
0 \\
\infty \\
\infty \\
-1\end{array}\right|$ & $\mid$\begin{tabular}{c|}
$\vec{\infty}$ \\
$\infty$ \\
0 \\
\hdashline \\
$\tilde{n}$ \\
$\mid$
\end{tabular} & 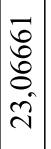 & 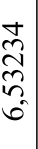 & 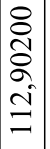 & 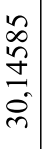 & $\begin{array}{l}0 \\
0 \\
0 \\
0 \\
0 \\
- \\
-\end{array}$ & 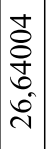 & 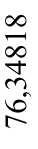 & $\begin{array}{l}8 \\
0 \\
0 \\
0 \\
n \\
\text { in }\end{array}$ \\
\hline 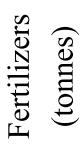 & $\begin{array}{l}= \\
\bar{\sigma} \\
\stackrel{\infty}{0}\end{array}$ & 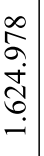 & 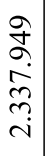 & $\begin{array}{l}0 \\
\infty \\
0 \\
\infty \\
\tilde{n} \\
\dot{\sigma} \\
\end{array}$ & 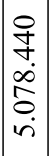 & $\begin{array}{l}\text { J } \\
- \\
8 \\
0 \\
0 \\
i\end{array}$ & 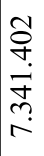 & 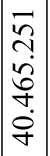 & $\begin{array}{l}\stackrel{\infty}{+} \\
\stackrel{+}{+} \\
\stackrel{0}{\circ} \\
\stackrel{+}{+}\end{array}$ & 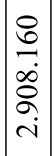 & 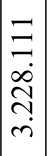 & $\left|\begin{array}{c|}\infty \\
0 \\
0 \\
\infty \\
0 \\
0 \\
\dot{c} \\
\end{array}\right|$ & 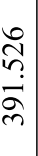 & 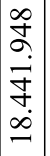 & 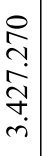 & 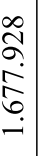 & 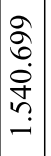 & & $\frac{\hat{m}}{2}$ \\
\hline 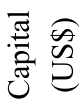 & $\begin{array}{c}0 \\
\infty \\
\stackrel{2}{c} \\
\end{array}$ & $\begin{array}{l}0 \\
0 \\
\dot{+} \\
\dot{m}\end{array}$ & $\begin{array}{l}\check{2} \\
\stackrel{\infty}{\dot{\infty}} \\
\end{array}$ & 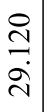 & $\begin{array}{l}0 \\
\infty \\
\infty \\
\infty \\
\infty \\
\infty\end{array}$ & $\begin{array}{l}\bar{\sigma} \\
\hat{\alpha}\end{array}$ & 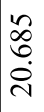 & $\left|\begin{array}{l}\infty \\
0 \\
0 \\
\infty \\
\infty \\
0 \\
0\end{array}\right|$ & $\begin{array}{c}\text { } \\
\tilde{\sigma} \\
\dot{0} \\
-\end{array}$ & 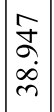 & $\begin{array}{l}\curvearrowright \\
\Xi \\
\Xi \\
\Xi\end{array}$ & $\mid \begin{array}{l}n \\
\tilde{b} \\
\dot{\infty} \\
\infty\end{array}$ & $\begin{array}{l}\stackrel{\infty}{\mathbb{N}} \\
\stackrel{\sim}{\sim}\end{array}$ & $\mid \begin{array}{l}\infty \\
\vec{J} \\
\dot{0} \\
\vec{e}\end{array}$ & $\begin{array}{l}\dot{8} \\
\dot{0} \\
\infty\end{array}$ & 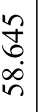 & 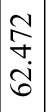 & & \begin{tabular}{l}
0 \\
\multirow{2}{0}{} \\
$\infty$ \\
-
\end{tabular} \\
\hline 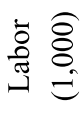 & $\left|\begin{array}{l}\tilde{N} \\
\widehat{\sim} \\
\text { in }\end{array}\right|$ & $\begin{array}{c}m \\
\stackrel{m}{-}\end{array}$ & 导 & $\begin{array}{l}\stackrel{\vartheta}{\rightleftharpoons} \\
\vec{\nabla}\end{array}$ & $\mid \begin{array}{l}\hat{\delta} \\
\dot{\delta} \\
\text { I }\end{array}$ & సิ & $\stackrel{\widetilde{Q}}{0}$ & $\begin{array}{l} \pm \\
\sigma \\
\infty \\
0 \\
0 \\
\infty\end{array}$ & $\begin{array}{l}\bar{\sigma} \\
\dot{n}\end{array}$ & $\mid \begin{array}{l}8 \\
0 \\
0 \\
\infty\end{array}$ & $\widehat{\mathbb{N}}$ & $\frac{\infty}{\infty}$ & $\frac{N}{\sim}$ & $\begin{array}{l}\tilde{N} \\
\hat{n} \\
\dot{\infty} \\
\sim\end{array}$ & 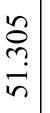 & 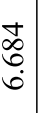 & \begin{tabular}{|c|}
$\infty$ \\
0 \\
0 \\
- \\
-
\end{tabular} & & 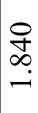 \\
\hline 通 & \begin{tabular}{|l}
$\overrightarrow{\vec{v}}$ \\
$\vec{\nabla}$
\end{tabular} & $\begin{array}{l}\stackrel{0}{n} \\
m \\
m \\
m\end{array}$ & $\mid \begin{array}{l}g \\
g \\
\dot{y} \\
f\end{array}$ & $\begin{array}{l}m \\
\dot{0} \\
a\end{array}$ & 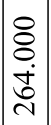 & 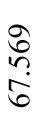 & 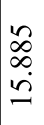 & 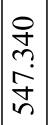 & $\begin{array}{l}\tilde{n} \\
\tilde{y} \\
\gamma\end{array}$ & $\left|\begin{array}{l}\tilde{\sim} \\
\tilde{n} \\
\tilde{n}\end{array}\right|$ & 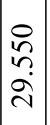 & \begin{tabular}{|l}
$\overrightarrow{\tilde{o}}$ \\
$\underline{\underline{z}}$ \\
\end{tabular} & $\begin{array}{c}\dot{W} \\
m \\
\infty \\
\infty\end{array}$ & $\mid \begin{array}{l}\infty \\
0 \\
\infty \\
2 \\
2 \\
=\end{array}$ & \begin{tabular}{l}
0 \\
\multirow{+}{*}{} \\
\multirow{2}{*}{} \\
\multirow{+}{*}{}
\end{tabular} & $\begin{array}{l}\overrightarrow{\tilde{\sigma}} \\
\dot{\varphi} \\
\vec{\sigma}\end{array}$ & 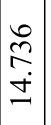 & & $\begin{array}{l}\vec{\infty} \\
\infty \\
-\end{array}$ \\
\hline 总 & 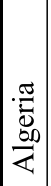 & 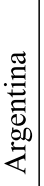 & 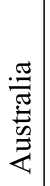 & E. & \begin{tabular}{|l|}
$\overline{\widetilde{N}}$ \\
$\overline{\mathscr{N}}$ \\
$\bar{m}$
\end{tabular} & ర్ & $\frac{0}{z}$ & 节 & है & 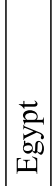 & 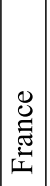 & 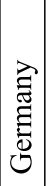 & 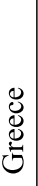 & 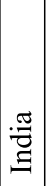 & 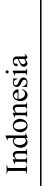 & ્ָ & $\frac{\vec{\pi}}{\mathbb{\Xi}}$ & 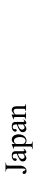 & 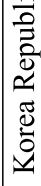 \\
\hline
\end{tabular}




\begin{tabular}{|c|c|c|c|c|c|c|c|c|c|c|c|c|c|c|c|c|c|c|c|c|c|}
\hline$\underline{\sim}$ & 0 & 0 & 0 & 0 & 0 & 0 & 0 & 0 & 0 & 0 & 0 & - & 0 & 0 & 0 & 0 & 0 & 0 & 0 & 0 & 0 \\
\hline $\mathscr{q}$ & 0 & 0 & 0 & 0 & - & 0 & 0 & 0 & 0 & 0 & 0 & 10 & 0 & 0 & 0 & 0 & 0 & 0 & 0 & 0 & 0 \\
\hline 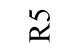 & 0 & 0 & 0 & 0 & 0 & 0 & 0 & 0 & 0 & 0 & 0 & 0 & 0 & 0 & 0 & 0 & 0 & - & 0 & 0 & 0 \\
\hline$\underset{\simeq}{ \pm}$ & 0 & 0 & - & 0 & 0 & 0 & 0 & 0 & 0 & - & 0 & 10 & - & 0 & 0 & 0 & 0 & 0 & 0 & 0 & 0 \\
\hline 2 & 0 & - & 0 & 0 & 0 & 0 & 0 & 0 & 0 & 0 & 0 & 10 & 0 & 0 & 0 & 0 & 0 & 0 & 0 & - & 0 \\
\hline$\approx \widetilde{Z}$ & 0 & 0 & 0 & - & 0 & 0 & - & - & - & 0 & - & 0 & 0 & 0 & - & - & - & 0 & - & 0 & 0 \\
\hline$\widetilde{\simeq}$ & - & 0 & 0 & 0 & 0 & - & 0 & 0 & 0 & 0 & 0 & 0 & 0 & - & 0 & 0 & 0 & 0 & 0 & 0 & - \\
\hline 宔 & $\mid \begin{array}{l}n \\
\infty \\
\infty \\
0\end{array}$ & $\mid \begin{array}{l}\overrightarrow{\widetilde{N}} \\
0 \\
0\end{array}$ & 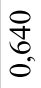 & $\begin{array}{l}\tilde{\xi} \\
\hat{\sigma}\end{array}$ & $\begin{array}{l}\tilde{2} \\
\tilde{n} \\
0\end{array}$ & $\begin{array}{l}\hat{\sigma} \\
\hat{0} \\
0\end{array}$ & $\begin{array}{l}\mathcal{W} \\
\infty \\
0 \\
0\end{array}$ & $\begin{array}{l}n \\
0 \\
\infty \\
0 \\
0\end{array}$ & $\begin{array}{l}\hat{\sigma} \\
\hat{0}\end{array}$ & 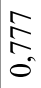 & $\begin{array}{l}\infty \\
\tilde{\sigma} \\
\hat{\sigma}\end{array}$ & $\begin{array}{l}0 \\
n \\
n \\
0\end{array}$ & $\frac{0}{\pi}$ & 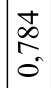 & $\frac{\tilde{n}}{\tilde{0}}$ & $\mid \begin{array}{l}\frac{1}{2} \\
0^{\prime}\end{array}$ & $\mid \begin{array}{l}9 \\
\dot{a} \\
0 \\
0\end{array}$ & $\begin{array}{l}\infty \\
0 \\
0 \\
0 \\
0\end{array}$ & $\begin{array}{l}0 \\
0 \\
0 \\
0\end{array}$ & $\begin{array}{l}\frac{ \pm}{\infty} \\
\vdots \\
0\end{array}$ & $\mid \begin{array}{l}\stackrel{2}{2} \\
\circ\end{array}$ \\
\hline 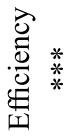 & $\mid \begin{array}{l}0 \\
\frac{8}{2} \\
0 \\
0\end{array}$ & $\begin{array}{c}2 \\
\delta \\
0 \\
\tilde{n} \\
0\end{array}$ & $\begin{array}{l}0 \\
\infty \\
+\infty \\
m \\
0 \\
0\end{array}$ & $\begin{array}{l}8 \\
8 \\
8 \\
-1\end{array}$ & 免 & 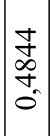 & 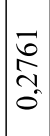 & $\begin{array}{l}0 \\
0 \\
0 \\
1 \\
1 \\
0\end{array}$ & 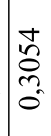 & $\stackrel{8}{\vdots}$ & \begin{tabular}{l}
$\tilde{\sigma}$ \\
\multirow{\sigma}{*}{} \\
0 \\
0
\end{tabular} & ஜ & 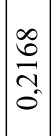 & 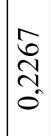 & 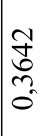 & $\stackrel{\infty}{\equiv}$ & $\begin{array}{l}n \\
\frac{n}{n} \\
0 \\
0\end{array}$ & 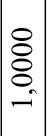 & $\begin{array}{l}\overline{0} \\
0 \\
0 \\
0\end{array}$ & $\begin{array}{l}8 \\
\vdots \\
0 \\
-\end{array}$ & 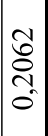 \\
\hline 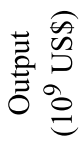 & $\begin{array}{l}\vec{\infty} \\
0 \\
0 \\
\tilde{o} \\
\sigma\end{array}$ & $\mid \begin{array}{l}0 \\
\infty \\
\infty \\
\infty \\
\tilde{\lambda}\end{array}$ & 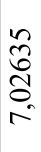 & $\left|\begin{array}{l}a \\
\hat{n} \\
5 \\
2 \\
a\end{array}\right|$ & $\mid \begin{array}{l}\infty \\
\tilde{n} \\
\infty \\
\infty \\
\sigma \\
-\end{array}$ & $\begin{array}{l}\overrightarrow{\mathcal{T}} \\
\mathbb{J} \\
\tilde{n} \\
\mathbb{\Xi}\end{array}$ & $\begin{array}{l}\infty \\
\tilde{N} \\
\hat{\infty} \\
\infty \\
\infty\end{array}$ & $\begin{array}{l}0 \\
0 \\
0 \\
n \\
n \\
0\end{array}$ & 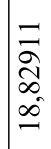 & $\begin{array}{l}\hat{\sigma} \\
\hat{\alpha} \\
\sigma\end{array}$ & 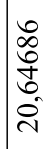 & 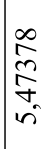 & 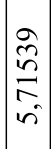 & 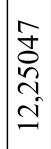 & $\frac{\stackrel{2}{2}}{\stackrel{N}{2}}$ & $\begin{array}{l}\sim \\
\infty \\
\tilde{n} \\
n \\
n\end{array}$ & 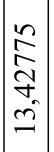 & 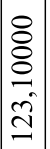 & $\begin{array}{l}\hat{\delta} \\
\delta \\
\hat{\sigma} \\
i \\
i\end{array}$ & \begin{tabular}{l}
$\frac{\pi}{\pi}$ \\
$\infty$ \\
\multirow{n}{n}{}
\end{tabular} & 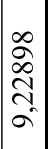 \\
\hline 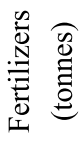 & $\mid \begin{array}{c}\bar{a} \\
\stackrel{2}{\tilde{n}} \\
\\
-i\end{array}$ & 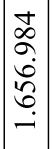 & 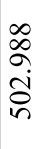 & $\begin{array}{l}\hat{\sigma} \\
\dot{o} \\
\dot{\alpha} \\
\hat{n}\end{array}$ & $\begin{array}{l}2 \\
\infty \\
\dot{8} \\
\stackrel{0}{2} \\
\dot{m}\end{array}$ & 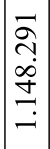 & $\begin{array}{l}8 \\
\stackrel{8}{0} \\
\infty \\
8 \\
0 \\
-1\end{array}$ & 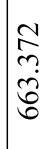 & 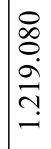 & 离 & $\begin{array}{l}\bar{n} \\
\tilde{n} \\
2 \\
2 \\
\end{array}$ & 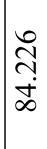 & 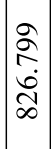 & 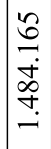 & $\begin{array}{l}\bar{n} \\
\infty \\
i \\
\sigma \\
i\end{array}$ & $\frac{\partial}{\dot{2}}$ & $\mid \begin{array}{c}0 \\
\infty \\
\infty \\
\\
\hat{n} \\
\infty \\
-\end{array}$ & 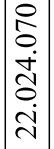 & $\begin{array}{l}8 \\
8 \\
\infty \\
\infty \\
1 \\
1\end{array}$ & 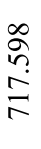 & $\begin{array}{l}8 \\
0 \\
\infty \\
\infty \\
\infty \\
0 \\
0 \\
-\end{array}$ \\
\hline 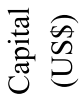 & $\mid \begin{array}{l}n \\
\infty \\
n \\
\stackrel{+}{\Xi} \\
=\end{array}$ & 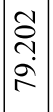 & 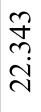 & $\begin{array}{l}\overrightarrow{8} \\
\grave{2} \\
i\end{array}$ & $\begin{array}{l}0 \\
\stackrel{0}{0} \\
0 \\
0\end{array}$ & $\mid \begin{array}{l}\stackrel{2}{\hat{D}} \\
\infty \\
\dot{\sim} \\
\end{array}$ & $\begin{array}{l}\hat{\sigma} \\
\hat{\sigma} \\
\hat{\sim}\end{array}$ & $\frac{\widetilde{v}}{\stackrel{v}{\gamma}}$ & $\begin{array}{l}\frac{m}{n} \\
\tilde{o} \\
\varrho\end{array}$ & $\begin{array}{l}\frac{1}{2} \\
\stackrel{i}{\circ}\end{array}$ & $\begin{array}{l}\stackrel{0}{2} \\
\stackrel{\sim}{\sim} \\
\dot{\sigma}\end{array}$ & $\frac{2}{\infty}$ & 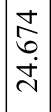 & $\frac{n}{\tilde{f}}$ & $\begin{array}{l}\overline{0} \\
m \\
\infty \\
\infty \\
\infty\end{array}$ & $\begin{array}{l}\vec{J} \\
\stackrel{2}{a}\end{array}$ & $\mid \begin{array}{l}\overrightarrow{2} \\
\stackrel{0}{0} \\
\dot{n}\end{array}$ & $\begin{array}{l}\frac{7}{ \pm} \\
\infty \\
\frac{0}{\delta}\end{array}$ & \begin{tabular}{l}
$\stackrel{+}{5}$ \\
\multirow{+}{*}{} \\
$\stackrel{+}{n}$
\end{tabular} & $\begin{array}{l}0 \\
\stackrel{n}{n} \\
\stackrel{n}{n}\end{array}$ & $\begin{array}{l}n \\
\infty \\
\infty \\
\infty \\
m\end{array}$ \\
\hline 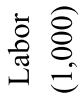 & 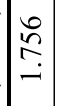 & $\begin{array}{c}\frac{1}{m} \\
\infty \\
\infty\end{array}$ & 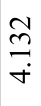 & $\stackrel{0}{\sim}$ & $\begin{array}{l}0 \\
\tilde{0} \\
\infty \\
\sim \\
\sim\end{array}$ & $\begin{array}{l}0 \\
\stackrel{0}{0} \\
\stackrel{2}{2} \\
-\end{array}$ & $\begin{array}{l}\stackrel{8}{\infty} \\
\infty \\
\infty \\
\dot{n}\end{array}$ & సે & 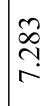 & $\overline{\widehat{\sigma}}$ & $\stackrel{n}{=}$ & $\stackrel{2}{\infty}$ & 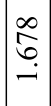 & 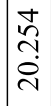 & $\begin{array}{l}5 \\
0 \\
-1\end{array}$ & 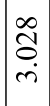 & $\hat{a}$ & $\begin{array}{l}n \\
\infty \\
\stackrel{2}{i} \\
i\end{array}$ & $\begin{array}{l}8 \\
\stackrel{2}{2} \\
i\end{array}$ & $\Re$ & 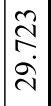 \\
\hline 吝 & $\mid$\begin{tabular}{l}
0 \\
$\infty$ \\
$\infty$ \\
\hdashline \\
\hdashline
\end{tabular} & $\begin{array}{c}8 \\
8 \\
0 \\
5 \\
0 \\
-\end{array}$ & $\begin{array}{l}\partial े \\
\grave{\partial} \\
\text { ते }\end{array}$ & $\overrightarrow{\widehat{\alpha}}$ & \begin{tabular}{l}
8 \\
$\vdots$ \\
\multirow{2}{*}{}
\end{tabular} & $\begin{array}{l}\stackrel{0}{n} \\
n \\
=\end{array}$ & 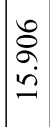 & $\begin{array}{l}\infty \\
\stackrel{ \pm}{ \pm}\end{array}$ & $\begin{array}{l}0 \\
0 \\
0 \\
i \\
\end{array}$ & 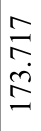 & 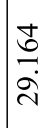 & $\begin{array}{l}\hat{n} \\
\infty \\
0 \\
\tilde{n}\end{array}$ & 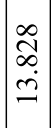 & $\begin{array}{l}8 \\
8 \\
0 \\
2\end{array}$ & 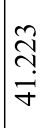 & 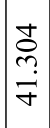 & 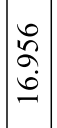 & 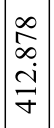 & 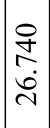 & $\begin{array}{l}\stackrel{R}{n} \\
\stackrel{n}{\sim}\end{array}$ & 苍 \\
\hline 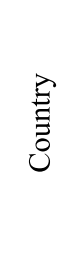 & 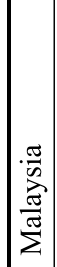 & 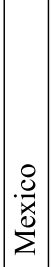 & $\begin{array}{l}0 \\
0 \\
0 \\
0 \\
0 \\
\Sigma\end{array}$ & 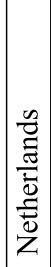 & 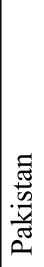 & 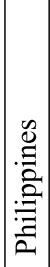 & 点 & 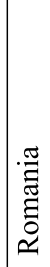 & |. & 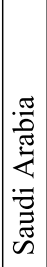 & 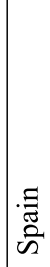 & 超 & 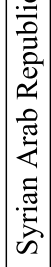 & 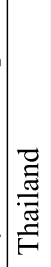 & 㞭 & 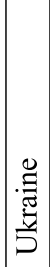 & 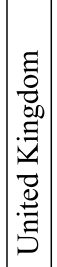 & 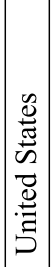 & 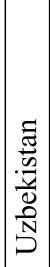 & 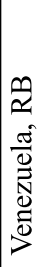 & 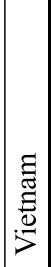 \\
\hline
\end{tabular}


continuous distribution concentrated on $\left(-V^{M}, V^{M}\right)$. The inefficiency error component is positive. It follows (2).

$$
\begin{aligned}
& y_{j}=g\left(x_{j}\right)+V^{M}-\left(V^{M}-v_{j}+u_{j}\right) \\
& y_{j}=\tilde{g}\left(x_{j}\right)-\varepsilon_{j}
\end{aligned}
$$

The component $\varepsilon_{j}$ is strictly positive. Following Banker (1993), Souza \& Staub (2007), and Banker \& Natarajan (2004, 2008), assuming, for example, a gamma family of distributions for the $\varepsilon_{j}$, it is possible to use DEA, output oriented and under variable returns to scale, to consistently estimate $\tilde{g}(x)$. Identical distributions are not required and one may let the mean $\mu$ of the inefficiency distribution be dependent on a linear function $\delta^{\prime} z$ of covariates or contextual variables. Following Simar \& Wilson (2007), we considered a two stage statistical model to estimate $\delta$ using only the inefficient firms. For this purpose, we fit a gamma distribution $\Gamma\left(p, \lambda_{j}\right)$ with mean $\mu_{j}=p / \lambda_{j}$, where $\lambda_{j}=\exp \left(-\delta^{\prime} z_{j}\right)$, by maximum likelihood, to DEA residuals $\hat{\varepsilon}_{j}=\left(\phi_{j}^{*}-1\right) y_{j}$. The empirical production function defined in (3), where the sup is restricted to vectors $\gamma$ for which $\sum_{j} \gamma_{j}=1$, consistently estimates $\tilde{g}(x)$ for $x \in K^{*}$. For input data points $x_{j}, \hat{g}\left(x_{j}\right)=\phi_{j}^{*} y_{j}$. The covariate of main concern here is HDI.

$$
\begin{aligned}
& \hat{g}(x)=\sup _{\gamma}\left\{\sum_{j} \gamma_{j} y_{j} ; \sum_{j} \gamma_{j} x_{j} \leq x, x \in K^{*}\right\} \\
& K^{*}=\left\{x \in K ; x \geq \sum_{j} \gamma_{j} x_{j}, \gamma_{j} \geq 0, \sum_{j} \gamma_{j}=1\right\}
\end{aligned}
$$

We obtain information on the constant $V^{M}$ assuming that the efficient units are producing on the technological frontier. In this context an optimum estimate would be $\hat{V}^{M}=\sum_{l=1}^{n_{l}} \hat{\mu}_{l} / n_{l}$, where $\hat{\mu}_{l}$ is the maximum likelihood estimate of $\mu_{l}$ and the sum is over the efficient units. The maximum likelihood estimate of $\mu_{l}$ is computed from the inefficient units. This is a subtle modification on the methods proposed by Banker \& Natarajan (2008). The use of the gamma distribution and the adaptation of the procedures of Simar \& Wilson (2007) is also original. In this context another possibility to model the inefficiency distribution would be given by the truncation at zero of the normal with mean $\mu_{j}$ and constant variance. This alternative did not fit well in our instance.

\section{EMPIRICAL RESULTS}

Table 1 shows the estimates of efficiency computed under the assumption of variable returns to scale. The nonparametric one sided test of Wilcoxon rank sum (Conover, 1998) point to marginal significance for the difference between the assumptions of variable and constant returns. For this reason, our choice was the less restrictive variable returns to scale model.

We notice that for each country $o$, the output oriented efficiency measurement is a solution of the linear programming $\operatorname{Max} \varphi$ subject to the restrictions $Y \gamma \geq \varphi y_{o}, X \gamma \leq x_{o}, \gamma \geq 0, \gamma 1=1$. The vector $\left(x_{o}, y_{o}\right)$ is the pair input-output for country $o$, and $X$ and $Y$ are the matrices formed with inputs and outputs for all countries in the analysis, respectively. Also $\hat{g}\left(x_{o}\right)=\varphi_{o}^{*} y_{o}$, where $\varphi_{o}^{*}$ is the solution of the linear programming problem. Efficiency quantities in Table 1 are inverted to bring their values to $(0,1]$. 
The distribution of efficiency scores depicted in Figure 1 has no outliers, but seems to have two models. The median efficiency is 0.466 . The first quartile is 0.298 and $30 \%$ of the countries are fully efficient.
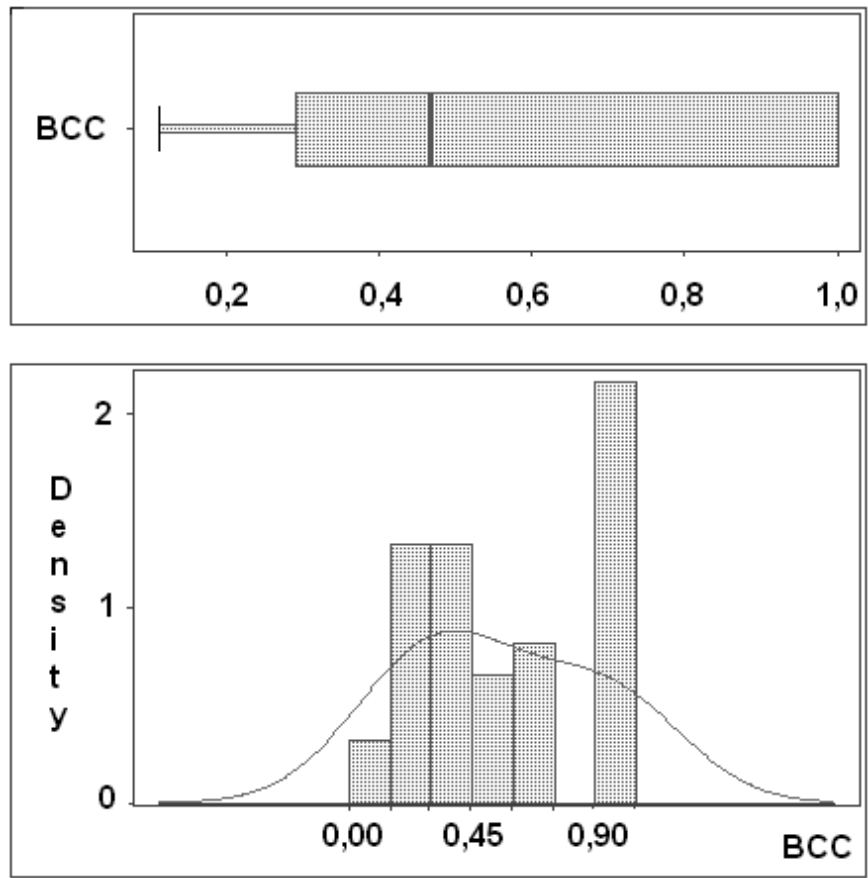

Figure 1 - Distribution of efficiency scores.

Some interesting considerations may be drawn from the efficiency scores in Table 1 (Annex). Among G-7 countries, France, Japan, USA and Canada are efficient, while UK, Italy and Germany show greater efficiency levels than the median. Other countries of high income, Netherlands, and middle income, Greece, Korea Republic, Venezuela and Saudi Arabia are efficient as well. At least, three countries of low income are also efficient: Algeria, China and Sudan.

Nine of the twenty countries with the largest output have efficiency scores lower than the median. These countries are Brazil, Indonesia, Turkey, Mexico, Pakistan, Russian Federation, Egypt, Iran and Argentina. This result suggests that the value added by the agricultural sector of these countries can be substantially improved reducing substantially the world agricultural output gap.

The gamma distribution fitted to non-efficient units produced Table 2. Regional dummies R5 and $\mathrm{R} 7$ were removed, since they are not represented in the regression. The base for the dummies R1$\mathrm{R} 4$ represented in the analysis is $\mathrm{R} 6$. We see that the coefficient - $\mathrm{b} 1$ is negative and statistically significant, indicating that an increase in HDI causes an increase in efficiency. The regional dummies coefficients indicate that R6 and R4 are equivalent and efficiently superior to the other three regions. 
Table 2 - Maximum likelihood estimates of inefficiency errors. Underlying gamma distribution has shape parameter $p$ and scale $\exp \left(-b_{0}-b_{1} x_{1}-b_{2} x_{2}-b_{3} x_{3}-b_{4} x_{4}-b_{5} x_{5}\right)$, where $x_{1}$ is the $\mathrm{HDI}, x_{2}$ is $\mathrm{R} 1, x_{3}$ is $\mathrm{R} 2, x_{4}$ is $\mathrm{R} 3, x_{5}$ is $\mathrm{R} 4$ and $b_{0}$ is $\mathrm{R} 6$.

\begin{tabular}{|l|c|c|c|c|c|}
\hline Parameter & Estimate & Error & $\mathrm{DF}$ & $t$ value & $\operatorname{Pr}>|t|$ \\
\hline$b_{0}$ (intercept) & -5.2817 & 1.0801 & 28 & -4.89 & $<0.0001$ \\
\hline$b_{1}$ (HDI) & 5.4182 & 1.8210 & 28 & 2.98 & 0.0060 \\
\hline$b_{2}$ (R1) & -1.3355 & 0.5915 & 28 & -2.26 & 0.0319 \\
\hline$b_{3}(\mathrm{R} 2)$ & -1.3691 & 0.6626 & 28 & -2.07 & 0.0482 \\
\hline$b_{4}$ (R3) & -1.3515 & 0.6556 & 28 & -2.06 & 0.0487 \\
\hline$b_{5}$ (R4) & -0.8676 & 0.5547 & 28 & -1.56 & 0.1290 \\
\hline$p$ & 2.9779 & 0.7555 & 28 & 3.94 & 0.0005 \\
\hline
\end{tabular}

Based on the maximum likelihood estimation and using efficient units, one obtains $\hat{V}^{M}=0.462$ with a standard error of 0.055 . United States and Canada were removed from this calculation since they were definite outliers for the values for which the $\hat{V}^{M}$ mean is computed.

In added values terms the agricultural sector could grow $53.9 \%$ using the available technology. Table 3 shows individual outputs and projections of potential outputs resulting from efficiency adjustments. It also shows the output gap. In absolute terms the median gap is $14,879 \times 10^{9}$ and the third quartile is $38,192 \times 10^{9}$. Pakistan and Malaysia are the leading relative contributors to potential increase in agricultural GDP since they are highly inefficient. Likewise other important countries are Mexico, Turkey and Indonesia.

Table 3 shows the output gap in $10^{9}$ dollar values. The upper quartile includes Pakistan, Malaysia, Mexico, Turkey, Indonesia, Iran, Brazil, Ukraine, Russian Federation and Thailand. This is an indication that these countries may increase substantially agricultural production with proper incentive policies.

\section{CONCLUSIONS}

This article assesses the efficiency of production for the major agricultural producers in the year of 2005. We estimated the output gap due to inefficiency for each economy and concluded that if these countries were working on the efficient frontier, the supply of agricultural GDP would increase by $53.9 \%$.

From the efficiency scores we depicted that among G-7 countries, only France, Japan, USA and Canada were efficient. Nine of the twenty countries with the largest output had efficiency scores lower than the median, including Brazil. This suggests that the value added by the agricultural sector can be improved by reducing the world agricultural output gap.

The regression analysis showed that an increase in HDI can cause an increase in agricultural efficiency. Regions R6 and R4 were equivalent and more efficient than the others in this model. 
Table 3 - Agricultural GDP: actual values, projections adjusted for efficiency, and absolute output gap $\left(\times 10^{9}\right.$ US\$).

\begin{tabular}{|c|c|c|c|}
\hline Country & Actual & Projection & Gap \\
\hline Algeria & 6,4694 & 6,4694 & 0 \\
\hline Argentina & 15,3570 & 33,1723 & 17,8153 \\
\hline Australia & 14,0113 & 19,4479 & 5,4367 \\
\hline Bangladesh & 13,1141 & 33,7776 & 20,6635 \\
\hline Brazil & 38,6614 & 84,9861 & 46,3247 \\
\hline Canada & 15,9097 & 15,9097 & 0 \\
\hline Chile & 5,7741 & 14,3489 & 8,5748 \\
\hline China & 215,5380 & 215,5380 & 0 \\
\hline Colombia & 10,6359 & 16,4183 & 5,7825 \\
\hline Egypt & 18,3006 & 44,4069 & 26,1064 \\
\hline France & 33,1088 & 33,1088 & 0 \\
\hline Germany & 23,0666 & 33,3850 & 10,3184 \\
\hline Greece & 6,5323 & 6,5323 & 0 \\
\hline India & 112,9020 & 120,8161 & 7,9141 \\
\hline Indonesia & 30,1459 & 80,1939 & 50,0481 \\
\hline Iran & 17,6081 & 65,7134 & 48,1054 \\
\hline Italy & 26,6400 & 41,5717 & 14,9316 \\
\hline Japan & 76,3482 & 76,3482 & 0 \\
\hline Korea Republic & 22,5000 & 22,5000 & 0 \\
\hline Malaysia & 9,2068 & 64,8295 & 55,6227 \\
\hline Mexico & 23,8181 & 76,3900 & 52,5719 \\
\hline Morocco & 7,0263 & 19,6938 & 12,6675 \\
\hline Netherlands & 9,5458 & 9,5458 & 0 \\
\hline Pakistan & 19,8452 & 81,6232 & 61,7781 \\
\hline Philippines & 14,3642 & 29,1905 & 14,8263 \\
\hline Poland & 8,8334 & 31,5263 & 22,6929 \\
\hline Romania & 6,5587 & 23,9546 & 17,3959 \\
\hline Russian Federation & 18,8291 & 61,1924 & 42,3633 \\
\hline Saudi Arabia & 9,9904 & 9,9904 & 0 \\
\hline Spain & 20,6469 & 42,8980 & 22,2511 \\
\hline Sudan & 5,4738 & 5,4738 & 0 \\
\hline Syrian Arab Republic & 5,7154 & 25,8988 & 20,1834 \\
\hline Thailand & 12,2505 & 53,5658 & 41,3154 \\
\hline Turkey & 29,1774 & 79,6445 & 50,4671 \\
\hline Ukraine & 5,5181 & 48,8845 & 43,3664 \\
\hline United Kingdom & 13,4277 & 18,3058 & 4,8780 \\
\hline United States & 123,1000 & 123,1000 & 0 \\
\hline Uzbekistan & 5,6991 & 33,8570 & 28,1579 \\
\hline Venezuela, RB & 5,1871 & 5,1871 & 0 \\
\hline Vietnam & 9,2290 & 44,2966 & 35,0676 \\
\hline
\end{tabular}


A possible implication for economic policy resulting from this article is that a way to minimize food scarcity in the world is reducing the inefficiency of the producing units of agricultural goods. Moreover, the statistical results also indicate that HDI is an important variable to increase agricultural efficiency. However, if on one hand an increase of HDI in producing units induces a decrease in inefficiency in agricultural production, and thus an increase in supply, on the other hand, the same increase of HDI, as a proxy for welfare of population, will increase the demand for food.

The net social benefits of the interaction between demand and supply in this context were not studied here. Further research is needed in this direction. However a startling conclusion is that there is space and technology to increase agricultural production in $53.9 \%$ without requiring additional resources.

\section{REFERENCES}

[1] Aвbott PC, Hurt C \& Tyner WE. 2008. What's driving food prices? Issue Report, Farm Foundation, July.

[2] Antunes Jlf, Peres MA, Mello TRD \& Waldman EA. 2006. Multilevel assessment of determinants of dental caries experience in Brazil. Community Dentistry and Oral Epidemiology, 34(2): 146-152.

[3] Arcelus FJ \& Arocena P. 2005. Productivity differences across OECD countries in the presence of environmental constraints. Journal of the Operational Research Society, 56: 1352-1362.

[4] Asian Development Bank. 2008. Food prices and inflation in developing Asia: Is poverty reduction coming to an end? Economics and Research Department. Manila, Philippines. Accessed online at http://www.adb.org/Documents/reports/food-prices-inflation/Flood-prices-Inflatio-pdf.

[5] BANKER RD \& NATARAJAN R. 2004. Statistical tests based on DEA efficiency scores. In: Handbook on Data Envelopment Analysis [edited by Cooper WW, Seiford LM \& Zhu J], Kluwer International Series, 299-321.

[6] BANKER RD \& NATARAJAN R. 2008. Evaluating contextual variables affecting productivity using data envelopment analysis. Operations Research, 56: 48-58.

[7] BANKER RD. 1993. Maximum likelihood, consistency and DEA: a statistical foundation. Management Science, 39(10): 1265-1273.

[8] Baptista AJMS, CAstro ER \& Teixeiras EC. 2004. Discriminação da função de produção, elasticidades de substituição de fatores na agropecuária do Estado de Minas Gerais. Revista de Economia, Agronegócio, 2(2): 161-182.

[9] Coelli TJ \& RAO DSP. 2005. Total factor productivity growth in agriculture: a Malmquist index analysis of 93 countries, 1980-2000. Agricultural Economics, 32: 115-134.

[10] Conover WJ. 1998. Practical Nonparametric Statistics. Wiley, New York.

[11] DAWE D. 2008. Have recent increases in international cereal prices been transmitted to domestic economies? The experience in seven large Asian countries. ESA Working paper No. 08-03. Food and Agriculture Organization of the United Nations (FAO), Rome. 
[12] Despotis DK. 2005a. A Reassessment of the Human Development Index via Data Envelopment Analysis. Journal of the Operational Research Society, 56(8): 969-980.

[13] Despotis DK. 2005b. Measuring human development via data envelopment analysis: The case of Asia and the Pacific. Omega, 33(5): 385-390.

[14] Dyson T. 1994. Population growth and food production: recent global and regional trends. Population and Development Review, 20(2): 397-411.

[15] Food and Agriculture Organization of the United Nations. 2008a. Growing demand on agriculture and rising prices of commodities: An opportunity for smallholders in low-income, agricultural-based countries? Paper prepared by the Trade, Markets, and Agricultural Development Economics Division of FAO for the Round Table, $31^{\text {st }}$ session of IFAD's Governing Council, Rome.

[16] Food and Agriculture Organization of the United Nations. 2008b. Food and Agriculture Organization of the United Nations. - Statistics. Accessed online at http://www.fao.org/corp/statistics/en/.

[17] Fulginiti LE \& PeRrin RK. 1997. LDC agriculture: Nonparametric Malmquist productivity indexes. Journal of Development Economics, 53(2): 373-390.

[18] Gomes EG. 2008. Uso de modelos DEA em agricultura: revisão da literatura. Engevista, 10: 27-51.

[19] Gorton M \& Davidova S. 2004. Farm productivity and efficiency in the CEE applicant countries: a synthesis of results. Agricultural Economics, 30(1): 1-16.

[20] HaYAmi Y \& RUtTAN V. 1970. Agricultural productivity differences among countries. The American Economic Review, 40: 895-911.

[21] Headey D, Alauddin M \& Rao DSP. 2010. Explaining agricultural productivity growth: an international perspective. Agricultural Economics, 41: 1-14.

[22] International Food Policy Research Institute. 2008. Global Food Crises. Monitoring and Assessing Impact to Inform Policy Responses. Food Policy Report. Accessed online at http://www.ifpri.org/pubs/fpr/pr19.pdf.

[23] IVANIC M \& MARTIN W. 2008. Implications of higher global food prices for poverty in low-income countries. Policy Research Working paper No. 4594. Development Research Group, World Bank, Washington.

[24] Kumar S, Russell RR. 2002. Technological change, technological catch-up, and capital deepening: relative contributions to growth convergence. The American Economic Review, 92(3): 527-548.

[25] Kutner M, Nachtsheim C, Neter J \& Li W. 2004. Applied Linear Statistical Models. $5^{\text {th }}$ Edition. United States: McGraw-Hill.

[26] Mahlberg B \& Obersteiner M. 2001. Remeasuring the HDi by Data Envelopment Analysis. International Institute for Applied Systems Analysis Interim Report 01-069.

[27] Maudos J, Pastor JM \& Serrano L. 1999. Total factor productivity measurement and human capital in OCDE countries. Economics Letters, 63: 39-44.

[28] Nicholson NK \& EsSEKS JD. 1978. The politics of food scarcities in developing countries. International Organization, 32(3): 679-719. 
[29] Nin A, Channing A \& Preckel PV. 2003. Is agricultural productivity in developing countries really shrinking? New evidence using a modified nonparametric approach. Journal of Development Economics, 71(2): 395-415.

[30] Nogueira MA. 2005. Eficiência técnica na agropecuária das microrregiões brasileiras. Tese (Doutorado em Economia Aplicada), Universidade Federal de Viçosa. 105p.

[31] Organization for Economic Co-operation and Development. 2008. Rising food prices: causes and consequences. May. Accessed online at http://www.oecdobserver.org/.

[32] Otsuki T, HARDiE IW \& REIS EJ. 2002. The implication of property rights for joint agriculturetimber productivity in the Brazilian Amazon. Environment and Development Economics, 7: 299-323.

[33] OXFAM International. 2008. Oxfam International position on food prices. April. Accessed online at http://www.oxfam.org/.

[34] Pereira MF, Silveira JST, Lanzer EA \& Samohyl RW. 2002. Productivity growth and technological progress in the Brazilian agricultural sector. Pesquisa Operacional, 22(2): 133-146.

[35] RAY SC \& DESLI E. 1997. Productivity growth, technical progress, and efficiency change in industrialized countries: comment. The American Economic Review, 87(5): 1033-1039.

[36] REIMER JJ \& KANG SM. 2010. Estimation of trade and domestic distortions: an application to world agriculture. Journal of Productivity Analysis (forthcoming).

[37] Rezitis AN. 2010. Agricultural productivity and convergence: Europe and the United States. Applied Economics, 42(8): 1029-1044.

[38] Rosegrant MW. 2008. Biofuels and grain prices: impacts and policy responses. IFPRI, Washington, May. Accessed online at http://www.ifpri.org/.

[39] Sharma S \& ThOMAS VJ. 2008. Inter-country R\&D efficiency analysis: An application of data envelopment analysis. Scientometrics, 76(3): 483-501.

[40] SIMAR L \& WILSON PW. 2007. Estimation and inference in two-stage, semi-parametric models of production processes. Journal of Econometrics, 136(1): 31-64.

[41] Souza GS \& Staub RB. 2007. Two-stage inference using data envelopment analysis efficiency measurements in univariate production models. International Transactions in Operational Research, 14: $245-258$.

[42] Souza GS, Gomes EG, Gazzola R \& Avila AFD. 2010. Economic efficiency and the effects of education and investment in research in the Brazilian agricultural sector. Anais do XLII Simpósio Brasileiro de Pesquisa Operacional, Bento Gonçalves.

[43] Thiam A, Bravo-Ureta BE \& Rivas TE. 2001. Technical efficiency in developing country agriculture: a meta-analysis. Agricultural Economics, 25: 235-243.

[44] Thirtle C, Piesse J, Lusigi A \& Suhariyanto K. 2003. Multi-factor agricultural productivity, efficiency and convergence in Botswana, 1981-1996. Journal of Development Economics, 71(2): 605-624.

[45] TyAGI P, YADAV SP \& Singh SP. 2009. Relative performance of academic departments using DEA with sensitivity analysis. Evaluating and Program Planning, 32: 168-177.

[46] United Nations Development Programme. 2006. Human Development Report. Beyond Scarcity: Power, Poverty and the Global Water Crisis. Palgrave Macmillan, New York. 
[47] VAldÉs A \& Foster W. 2008. Do high world commodity prices hurt or help family farms? A simulation case study for Chile. Paper presented at the FAO Expert Workshop on Policies for the Effective Management of Sustained Food Price Increases, Rome, July.

[48] Vicente JR. 2004. Mudança tecnológica, eficiência, produtividade total de fatores na agricultura brasileira, 1970-95. Economia Aplicada, 8(4): 729-760.

[49] Von Braun J, Ahmed A, Asenso-Okyere K, Fan S, Gulati A, Hoddinott J, PandyaLorch R, Rosegrant MW, Ruel M, Toreno M, van Rheenen T \& von Grebmer K. 2008. High food prices: The what, who, and how of proposed policy action. IFPRI, Washington.

[50] WORLD BANK. 2008a. Rising food prices: policy options and World Bank response. World Bank. Accessed online at http:/siteresources.worldbank.org/.

[51] World Bank. 2008b. Double jeopardy: Responding to high food and fuel prices. G8 HokkaidoToyako Summit, July. Accessed online at http://www.worldbank.org/html/extdr/foodprices/.

[52] World BANK. 2008c. Data-World Development Indicators 2008. Accessed online at http://publications.worldbank.org/GDF/.

[53] World ECONOMic Forum. 2008. Global risks 2008: a global risk network report. January. Accessed online at http://www.weforum.org/. 